\title{
ON THE GENERALIZED NAKAYAMA CONJECTURE AND THE CARTAN DETERMINANT PROBLEM
}

\author{
BY
}

\author{
K. R. FULLER ${ }^{1}$ AND B. ZIMMERMANN-HUISGEN
}

\begin{abstract}
For Artin algebras allowing certain filtered module categories, the Generalized Nakayama Conjecture is shown to be true; our result covers all positively graded Artin algebras and those whose radical cube is zero. For the corresponding class of left artinian rings we prove that finite global dimension forces the determinant of the Cartan matrix to be 1 .
\end{abstract}

1. Introduction. In 1958 Nakayama conjectured that any finite dimensional algebra of infinite dominant dimension is quasi-Frobenius. A positive solution could be considered a step towards the-stronger-finitistic dimension conjecture, claiming that all finite dimensional algebras have finite finitistic dimension (see e.g. [9, p. 105] for details). Another problem arose from Nakayama's conjecture, namely the Generalized Nakayama Conjecture (GNC) as proposed by Auslander and Reiten $[\mathbf{1}]$ : it suggests that, given an Artin algebra $R$, every indecomposable injective (left) $R$-module appears (up to isomorphism) as a direct summand of some term of the minimal injective resolution of ${ }_{R} R$. The validity of (GNC) implies that of the Nakayama Conjecture; given some injective resolution of ${ }_{R} R$ which consists entirely of projective modules, the minimal injective resolution will have the same property, whence (GNC) entails that all injective modules are projective.

A further, unrelated investigation turns out to profit from the technique we use to tackle (GNC), the question being whether for a left artinian ring of finite global dimension the determinant of the Cartan matrix is necessarily 1.

What is known?

As for the Nakayama Conjecture, Mueller and Morita and Tachikawa $[8,7]$ provided a positive answer for QF-3 algebras whose minimal faithful left ideals have endomorphism rings of finite representation type. Recently, Wilson [10] gave a proof for (GNC) in case $R$ is a positively graded finite dimensional algebra over a field. Our first major goal will be to establish (GNC) for all Artin algebras $R$ which are either positively graded or satisfy $J^{3}=0$, where $J$ is the Jacobson radical of $R$. Both situations (and others) arise as special cases of a more general statement (Theorem A). Even though the core of our argument, inspired by a trick of Eilenberg's, is essentially different from Wilson's, we owe a great debt to the ideas developed by Wilson in [10].

Concerning the above problem on the Cartan matrix $C$ of a left artinian ring $R$, Eilenberg [4] showed that finite left global dimension of $R$ entails $\operatorname{det} C=$ \pm 1 . Zacharia [11] secured $\operatorname{det} C=1$ whenever $\operatorname{lgl} \operatorname{dim} R \leq 2$, while, for left

Received by the editors March 19, 1985.

1980 Mathematics Subject Classification. Primary 16A35, 16A64; Secondary 16A03, 16A60.

${ }^{1}$ The first author faithfully acknowledges the support of the University of Hawaii during part of this project. 
serial rings $\operatorname{det} C=1$ is even equivalent to finite left global dimension according to Burgess, Voss and the authors [2]. Here we will modify an approach of Wilson [10] who obtained $\operatorname{det} C=1$ for all positively graded finite dimensional algebras of finite global dimension. It proves useful to consider a refined Cartan matrix which, being no more difficult to compute than the classical one, stores considerably more information. With its aid we will simultaneously answer the determinant question for left artinian rings with either a positive grading or $J^{3}=0$, as well as for left serial rings (Theorem $\mathrm{B}$ ).

We conclude with an application of our method to a computation of $\operatorname{Ext}^{1}(S, S)$, where $S$ is a simple module over an artinian ring.

Throughout, $R$ will be a left artinian ring with identity and $e_{1}, \ldots, e_{n}$ a complete set of orthogonal primitive idempotents in $R$. Since in none of the problems at stake will we lose generality by restricting our attention to basic rings, we will assume that the left ideals $R e_{i}, 1 \leq i \leq n$, are pairwise nonisomorphic.

2. Filtered $R$-categories and prototypical examples. Suppose that $A$ is a skeletally small abelian category such that each object of $A$ has an underlying structure of a finitely generated left $R$-module and all morphisms in $A$ are $R$-linear. Moreover, we require that addition and composition of morphisms in $A$ coincide with the corresponding operations for $R$-linear maps. Denote the forgetful functor $A \rightarrow R$-mod by $\psi$ (here $R$-mod stands for the category of all finitely generated left $R$-modules).

A category $A$ as above together with a family $\left(F^{k}\right)_{k \in \mathbf{Z}}$ of additive subfunctors of the identity functor on $A$ is called a filtered $R$-category if the following conditions are satisfied:

(F1) An object $A$ in $A$ is simple, indecomposable, projective or injective if and only if $\psi(A)$ is such in $R$-mod.

(F2) The functor $\psi$ is exact and preserves injective envelopes as well as projective covers (whenever they exist in $A$ ).

(F3) $F^{k} A \supseteq F^{k+1} A$ and the quotient $F^{k} A / F^{k+1} A$ is (zero or) a direct sum of simple objects for each $A$ in $A$ and all $k \in \mathbf{Z}$. Furthermore, there are integers $u$ and $v$ such that $F^{u} A=A$ and $F^{v} A=0$ (call the largest $c$ with $F^{c} A=A$ the codegree of $A$, the largest $d$ with $F^{d} A \neq 0$ the degree of $A$, and write $c=\operatorname{codeg} A$, resp. $d=\operatorname{deg} A$ ).

(F4) Given any two indecomposable projective modules $P$ and $Q$ in $A$ with $\psi(P) \cong \psi(Q)$, there exists an integer $l$ such that

$$
\psi\left(F^{k} P / F^{k+1} P\right) \cong \psi\left(F^{k+l} Q / F^{k+l+1} Q\right)
$$

for all $k \in \mathbf{K}$.

(F5) A contains objects $S_{1}, \ldots, S_{n}$ of codegree zero such that each simple left $R$-module is isomorphic to exactly one of the modules $\psi\left(S_{1}\right), \ldots, \psi\left(S_{n}\right)$. Moreover, each $S_{i}$ has a projective cover $P_{i}$ in $A$.

We say that $R$ is $A$-filtered if $R$ gives rise to a filtered $R$-category $A$. Moverover, we will often write $S_{i}$ for $\psi\left(S_{i}\right)$ and $P_{i}$ for $\psi\left(P_{i}\right)$ when there is no danger of confusion. 
Examples of filtered categories. 1. The category $A=R$-mod together with the "radical filtration", i.e. with the functors

$$
\begin{aligned}
& F^{k} A=J^{k} A \text { for } k \in \mathbf{Z}, k \geq 0, \\
& F^{k} A=A \text { for } k \in \mathbf{Z}, k \leq 0
\end{aligned}
$$

is a filtered $R$-category.

2. The category $A=R$-mod is once more a filtered $R$-category when equipped with the following "socle filtration": $F^{k} A=0$ for $k>0$, and $F^{k} A$ is the preimage in $A$ of the socle of $A / F^{k+1} A$ for $k \leq 0$.

3. Suppose that $R$ is positively graded, that is $R$ is a direct sum of abelian groups

$$
R=\bigoplus_{k=0}^{\infty} R_{k} \quad \text { with } R_{i} R_{j} \subseteq R_{i+j}
$$

such that $J=\bigoplus_{k=1}^{\infty} R_{k}$. Then the category $A=R$-gr of all finitely generated graded left $R$-modules (here negative gradings are allowed: $A=\bigoplus_{k \in \mathbf{Z}} A_{k}$ with abelian groups $A_{k}$ such that $R_{i} A_{j} \subseteq A_{i+j}$ ) together with the degree zero homomorphisms is a filtered $R$-category with the following functors:

$$
F^{k} A=\bigoplus_{l \geq k} A_{l} \quad \text { if } A=\bigoplus_{l \in \mathbf{Z}} A_{l} .
$$

We refer to [3 and 5] for information on graded artinian rings and Artin algebras.

We start by assembling a few elementary facts for futher reference.

LEMMA 1. Let $A$ be a filtered $R$-category and $A$ an object of codegree $c$ and degree $d$ :

(1) $\psi$ preserves direct sums; in particular, $A$ is semisimple if and only if the same is true for $\psi(A)$ in $R$-mod.

(2) $J \psi(A) \subseteq \psi\left(F^{c+1} A\right)$.

(3) $\psi\left(F^{d} A\right) \subseteq \operatorname{Soc}(\psi(A))$.

(4) If $f: A \rightarrow B$ is an epimorphism in $A$, then $\operatorname{codeg} A \leq \operatorname{codeg} B$.

(5) If $f: A \rightarrow B$ is a monomorphism in $A$, then $\operatorname{deg} A \leq \operatorname{deg} B$.

Proof. (1) is clear. For (2) observe that by (F2) and (F3)

$$
\psi\left(A / F^{c+1} A\right)=\psi\left(F^{c} A / F^{c+1} A\right) \cong \psi\left(F^{c} A\right) / \psi\left(F^{c+1} A\right)
$$

is a semisimple $R$-module. An analogous argument shows (3). Statement (4) follows immediately from the fact that $B=f\left(F^{c} A\right) \subseteq F^{c} B$, while (5) is again symmetric.

3. A filtered Grothendieck module for a filtered $R$-category. Starting with a filtered $R$-category $A$, we follow the Grothendieck pattern in defining a module $\mathcal{G}(A)$ over the ring $\mathbf{Z}((T))$ of integral Laurent series. Our goal is to identify the objects of $A$ with their weighted composition factors, where the weight takes the layering through the functors $F^{k}$ into account. More precisely, let

$$
G(A)=F(A) / R(A)
$$


as follows: $\mathcal{F}(\mathcal{A})$ is the free $\mathbf{Z}((T))$-module which has as a basis the set $|A|$ of isomorphism types of objects of $A$, and $R(A)$ is the $\mathbf{Z}((T))$-submodule generated by the elements

$$
D(A)=A-\sum_{k \in \mathbf{Z}} \sum_{i=1}^{n} n_{i k} T^{k} S_{i}
$$

where $A \in|A|$ and

$$
\psi\left(F^{k} A / F^{k+1} A\right) \cong \bigoplus_{i=1}^{n} S_{i}^{n_{i k}}
$$

for each $k \in \mathbf{Z}$. Denote the class of $A$ in $\mathcal{G}(A)$ by $[A]$ and make the following elementary observations:

LEMMA 2. (1) $\left[S_{1}\right], \ldots,\left[S_{n}\right]$ is a free basis for $g(A)$.

(2) $[A \oplus B]=[A]+[B]$.

(3) If $P$ and $Q$ are indecomposable projective objects in $A$ of codegree $p$, resp. $q$, then $[P]=T^{p-q}[Q]$, provided that $\psi(P) \cong \psi(Q)$ in $R$-mod.

Proof. (1) From the definition of $R(A)$ it is clear that $\left[S_{1}\right], \ldots,\left[S_{n}\right]$ span $\mathcal{G}(\mathcal{A})$. To show linear independence, suppose that $g_{i}(T), h_{j}(T)$ are elements of $\mathbf{Z}((T))$ and $A_{j} \in|A|$ with

$$
\begin{aligned}
\sum_{i=1}^{n} g_{i}(T) S_{i} & =\sum_{j=1}^{m} h_{j}(T) D\left(A_{j}\right) \\
& =\sum_{j=1}^{m} h_{j}(T)\left(A_{j}-\sum_{k \in \mathbf{Z}} \sum_{i=1}^{n} n_{i k}^{(j)} T^{k} S_{i}\right)
\end{aligned}
$$

in $\mathcal{F}(\mathcal{A})$. Since $D\left(S_{i}\right)=S_{i}-S_{i}=0$, we may assume that none of the $A_{j}$ is isomorphic to any of the $S_{i}$ in $A$. But then $h_{j}(T)=0$ for $j=1, \ldots, m$, and consequently $g_{i}(T)=0$ for $i=1, \ldots, n$. Part (2) is valid because the functors $F^{k}$ commute with direct sums, and (3) follows from (F4).

In case the simple objects $S_{i}$ have injective envelopes in $A$, the classes of the latter generate a submodule of rank $n$ in $\mathcal{G}(\mathcal{A})$. Namely

LEMMA 3. Suppose that $E_{i}$ is an injective envelope of $S_{i}$ in $A$ for $i=1, \ldots, n$. Then the elements $\left[E_{1}\right], \ldots,\left[E_{n}\right]$ of $\mathcal{G}(\mathcal{A})$ are linearly independent over $\mathbf{Z}((T))$.

PROOF. Expand each $\left[E_{j}\right]$ in terms of the basis $\left[S_{1}\right], \ldots,\left[S_{n}\right]$, say

$$
\left[E_{j}\right]=\sum_{i=1}^{n} l_{i j}\left[S_{i}\right]
$$

with $l_{i j} \in \mathbf{Z}((T))$ and set $L=\left(l_{i j}\right)_{1 \leq i, j \leq n}$. All we have to show is that $\operatorname{det} L \neq 0$; for then $L$ is an invertible matrix over the field of fractions of $\mathbf{Z}((T))$.

Note that the $l_{i j}=\sum_{k} l_{i j}^{(k)} T^{k}$ are actually polynomials in $T$ and $T^{-1}$, where $l_{i j}^{(k)}$ is the number of occurrences of the simple $R$-module $S_{i}$ in $\psi\left(F^{k} E_{j} / F^{k+1} E_{j}\right)$. Setting $d_{j}=\operatorname{deg} E_{j}$, we have $\psi\left(F^{d_{j}} E_{j}\right)=S_{j}=\operatorname{Soc}\left(\psi\left(E_{j}\right)\right)$ (compare Lemma $1(3)$ ) and $F^{k} E_{j}=0$ for $k>d_{j}$. Hence the monomial of highest degree in $T$ appearing in $l_{j j}$ is $T^{d_{j}}$, whereas all the polynomials $l_{i j}=l_{i j}\left(T, T^{-1}\right)$ which are found in the 
same column of $L$ have degrees in $T$ strictly below $d_{j}$. If we expand $\operatorname{det} L$ as the sum of signed elementary products of the entries in $L$, it thus becomes clear that

$$
\operatorname{det} L=T^{d_{1}+\cdots+d_{n}}+q\left(T, T^{-1}\right),
$$

where $q\left(T, T^{-1}\right)$ has degree strictly below $d_{1}+\cdots+d_{n}$ in $T$. This ensures that $\operatorname{det} L \neq 0$.

4. Morphisms which respect the filtration and examples revisited. Our strategy in proving (GNC) for rings with self-duality and a suitable filtered $R$ category $A$ will be to prove the dual $(\mathrm{GNC})^{*}$ via $A$ : Given that each $S_{j}$ has an injective envelope $E_{j}$ in $\mathcal{A}$, and that each $E_{j}$ has in turn a minimal projective resolution

$$
0 \leftarrow E_{j} \leftarrow Q_{0 j} \leftarrow Q_{1 j} \leftarrow \cdots
$$

in $A$, we want to show that each $\psi\left(P_{k}\right)$, i.e. each $R e_{k}$, occurs (up to $R$-isomorphism) as an $R$-direct summand of some $\psi\left(Q_{i j}\right)$. The advantage of shifting the problem to $A$ lies in the fact that, having taken some additonal precautions, we will be able to expand the $\left[E_{j}\right]$ in $\mathcal{G}(\mathcal{A})$ as $\mathbf{Z}((T))$-linear combinations of those $\left[P_{k}\right]$ 's whose $R$ isomorphism classes appear in some term along the above resolution and then invoke Lemma 3. The additional technical requirement is that the linking morphisms in the resolutions at stake not tangle the filtration of the objects too seriously.

Let $\mathcal{A}$ be a filtered $R$-category with filtration $\left(F^{k}\right)_{k \in \mathbf{Z}}$ and let $f: A \rightarrow B$ be a morphism in $A$. We say that $f$ respects the filtration in case the following three conditions are satisfied:

(R1) $f\left(F^{k} A\right)=F^{k} B$ for all $k \in \mathbf{Z}$ (so, in particular, $f$ is an eipmorphism).

(R2) $\operatorname{codeg}(\operatorname{Ker} f) \geq \operatorname{codeg} A$.

(R3) There exists a decomposition of $K=\operatorname{Ker} f$

$$
K=K_{1} \oplus \cdots \oplus K_{r}
$$

and nonnegative integers $u_{1}, \ldots, u_{r}$ such that

$$
\psi\left(K \cap F^{k} A / K \cap F^{k+1} A\right) \cong \bigoplus_{i=1}^{r} \psi\left(F^{k-u_{i}} K_{i} / F^{k-u_{i}+1} K_{i}\right)
$$

in $R$-mod.

REMARK. Note that whenever $f: A \rightarrow B$ respects the filtration, then any refinement of a decomposition of $K=\operatorname{Ker} f$ as in (R3) will again satisfy (R3). In particular, (R3) will hold for any indecomposable decomposition of $K$.

The following two lemmas will smooth the terrain for our main theorem.

LEMMA 4. If $f: A \rightarrow B$ respects the filtration, then the following equality holds in $G(A)$ :

$$
[B]=[A]-\sum_{i=1}^{r} T^{u_{i}}\left[K_{i}\right]
$$

where the $K_{i}$ and $u_{i}$ are as in (R3).

Proof. For any $X \in|A|$ set

$$
X^{(k)}=T^{k} \sum_{i=1}^{n} n_{i k}\left[S_{i}\right] \quad \text { if } \psi\left(F^{k} X / F^{k+1} X\right) \cong \bigoplus_{i=1}^{n} S_{i}^{n_{i k}},
$$


so that $[X]=\sum_{k \in \mathbf{Z}} X^{(k)}$. Then, observing that $f$ induces a split exact sequence

$$
0 \rightarrow\left(K \cap F^{k} A / K \cap F^{k+1} A\right) \rightarrow\left(F^{k} A / F^{k+1} A\right) \rightarrow\left(F^{k} B / F^{k+1} B\right) \rightarrow 0
$$

in $R$-mod (we dropped $\psi$ for better legibility), we see that

$$
A^{(k)}=B^{(k)}+\sum_{i=1}^{r} T^{u_{i}} K_{i}^{\left(k-u_{i}\right)}
$$

by the definition of the $K_{i}$ and $u_{i}$. Summation over $k \in \mathbf{Z}$ yields

$$
[A]=[B]+\sum_{i=1}^{r} T^{u_{i}}\left[K_{i}\right] .
$$

Given a nonzero integer polynomial $h=h\left(T, T^{-1}\right)$ in $T$ and $T^{-1}$, we call the exponent of the highest monomial in $T$ that occurs nontrivially in $h$ the degree of $h$, the lowest among the exponents of $T$ the codegree of $h$. Set $\operatorname{deg} h=\operatorname{codeg} h=\infty$ for $h=0$.

LEMMA 5. Let $f: A \rightarrow B$ be a morphism in $A$ which respects the filtration and whose kernel $K$ is contained in the radical of $A$ (as an $R$-module). Then the following is true:

(1) $\operatorname{codeg} A=\operatorname{codeg} B$.

(2) If $K=\bigoplus_{j=1}^{r} K_{j}$ with integers $u_{1}, \ldots, u_{r} \geq 0$ as in (R3), then

$$
\operatorname{codeg} K_{j}+u_{j} \geq \operatorname{codeg} B+1
$$

for all $j=1, \ldots, r$.

(3) Further specification of $f$ to be a projective cover of $B$ yields

$$
[B]=\sum_{i=1}^{n} h_{i}\left[P_{i}\right]-\sum_{j=1}^{r} T^{u_{j}}\left[K_{j}\right]
$$

with polynomials $h_{i}=h_{i}\left(T, T^{-1}\right)$ in $T$ and $T^{-1}$ such that

(i) $h_{i}=0$ if $P_{i}$ is not $R$-isomorphic to a direct summand of $\psi(A)$, and

(ii) $\operatorname{codeg} h_{i} \geq \operatorname{codeg} B$.

Proof. Denote the codegree of $A$, resp. $B$, by $a$, resp. $b$.

(1) From Lemma 1 we know that $a \leq b$. For the reverse inequality observe that $F^{a+1} B=f\left(F^{a+1} A\right) \neq B$, since $F^{a+1} A \neq A$ and Ker $f$ is superfluous in $A$. Thus $b \leq a$.

(2) Since codeg $K_{j} \geq \operatorname{codeg} K \geq a$, the latter inequality being (R2), and since $u_{j}$ is nonnegative, it will suffice to show that $u_{j}=0$ implies codeg $K_{j} \geq a+1$. So let $u_{j}=0$ and note that the quotient $K \cap F^{k} A / K \cap F^{k+1} A$ vanishes for all $k \leq a$. For $k<a$ this is clear, for $k=a$ it follows from the fact that $K \subseteq F^{a+1} A$ (compare Lemma 1). Now (R3) tells us that the quotient $\psi\left(F^{k} K_{j} / F^{k+1} K_{j}\right)=$ $\psi\left(F^{k-u_{j}} K_{j} / F^{k-u_{j}+1} K_{j}\right)$ is contained in the above quotient up to isomorphism, whence $F^{k} K_{j}=F^{k+1} K_{j}$ for all $k \leq a$. But the latter means codeg $K_{j} \geq a+1$.

(3) Decompose $A$ into indecomposable summands

$$
A=\bigoplus_{i=1}^{n} \bigoplus_{l=1}^{m_{i}} P_{i l}
$$


where each $\psi\left(P_{i l}\right)$ is isomorphic to $\psi\left(P_{i}\right) \cong R e_{i}$ in $R$-mod. Setting $p_{i l}=\operatorname{codeg} P_{i l}-$ $\operatorname{codeg} P_{i}$, Lemma 2 yields $\left[P_{i l}\right]=T^{p_{i l}}\left[P_{i}\right]$, and therefore $[A]=\sum_{i=1}^{n} h_{i}\left[P_{i}\right]$ with $h_{i}=\sum_{l=1}^{m_{i}} T^{p_{i l}}$. In view of Lemma 4 , we thus obtain

$$
[B]=\sum_{i=1}^{n} h_{i}\left[P_{i}\right]-\sum_{j=1}^{r} T^{u_{j}}\left[K_{j}\right] .
$$

Our construction guarantees that $h_{i}=0$ in case $m_{i}=0$. If, on the other hand, $h_{i} \neq 0$, then codeg $h_{i} \geq b$ because $p_{i l} \geq \operatorname{codeg} P_{i l} \geq a=b$, the first inequality following from the fact that $\operatorname{codeg} P_{i} \leq 0$ by Lemma 1 .

Standard examples. 1. Let $R$ be arbitrary left artinian, $A=R$-mod with the radical filtration (compare $\S 2$ ). Every epimorphism in $A$ satisfies (R1) and (R2).

(a) If $J^{3}=0$, then every epimorphism $f: A \rightarrow B$ in $A$ with Ker $f \subseteq J A$ respects the (radical) filtration.

PROOF. To verify (R3), observe first that any finitely generated indecomposable left $R$-module $M$ with $J^{2} M=0$ is either simple or satisfies Soc $M=J M$. Applying this to the indecomposable summands of $K=\operatorname{Ker} f$, we can find an indecomposable decomposition

$$
K=K_{1} \oplus \cdots \oplus K_{r}
$$

such that for some $1 \leq a \leq b \leq r+1$ we have

$$
\begin{array}{ll}
J K_{i} \neq 0 & \text { for } 1 \leq i<a, \\
J K_{i}=0 \text { and } \bigoplus K_{i} \cap J^{2} A=0 & \text { for } a \leq i<b, \\
J K_{i}=0 \text { and } K_{i} \subseteq J^{2} A & \text { for } b \leq i \leq r .
\end{array}
$$

Since $J K_{i}=\operatorname{Soc} K_{i}=J^{2} A \cap K_{i}$ for $1 \leq i<a$, one easily checks that the required $u_{i}$ are $u_{i}=1$ for $1 \leq i<b$ and $u_{i}=2$ for $b \leq i \leq r$.

(b) Now suppose that $R$ is left serial, meaning that each indecomposable projective $R$-module $R e_{i}$ is uniserial (i.e. has precisely one composition series), and endow $A=R$-mod with the radical filtration. Then all epimorphisms between uniserial modules respect the filtration (and so do all finite direct sums of such epimorphisms).

ProOF. Given an epimorphism $f: A \rightarrow B$ with $A$ and $B$ uniserial, let $u$ be the length of and check that this shifting constant (R3) is satisfied.

2. Given a positively graded ring $R$, consider $A=R$-gr with the filtration of $\S 2$ and observe that every epimorphism in $A$ respects the filtration (for (R3) choose $r=1$ and $u=u_{1}=0$ ).

5. The Nakayama conjecture. We call $R$ Nakayama filtered if $R$ allows a filtered $R$-category $A$ with the following properties: $A$ contains injective envelopes $E_{1}, \ldots, E_{n}$ of the simple objects $S_{1}, \ldots, S_{n}$ and each $E_{i}$ has a minimal projective resolution

$$
0 \leftarrow E_{i} \stackrel{f_{i 0}}{\longleftarrow} Q_{i 0} \stackrel{f_{i 1}}{\longleftarrow} Q_{i 1} \leftarrow \cdots
$$

in $A$ such that all direct summands of the maps $f_{i j}: Q_{i j} \rightarrow \operatorname{Im}\left(f_{i j}\right)$ respect the filtration. (Here a morphism $g_{1}: A_{1} \rightarrow B_{1}$ is called a direct summand of $g: A \rightarrow B$ if $g=g_{1} \oplus g_{2}: A_{1} \oplus A_{2} \rightarrow B_{1} \oplus B_{2}$ for some $g_{2}: A_{2} \rightarrow B_{2}$.)

$\S 4$ supplied us with major classes of examples: Whenever all indecomposable injective left $R$-modules are finitely generated and $R$ satisfies $J^{3}=0$ or is positively graded, $R$ is Nakayama filtered. Furthermore, all serial rings (i.e. rings which are left and right serial) are Nakayama filtered. 
THEOREM A. The dual of the generalized Nakayama conjecture holds for all Nakayama filtered rings. In more explicit terms: If $R$ is Nakayama filtered, then every indecomposable projective left $R$-module appears as a direct summand of some term in the minimal projective resolution of at least one indecomposable injective module.

COROLLARY. Both the Generalized Nakayama Conjecture and its dual hold for all Artin algebras with either radical cube zero or a positive grading.

PROOF. All we have to note is that Artin algebras possess self-dualities, which, in particular, ensures that their indecomposable injective modules are finitely generated.

The case where $R$ is a positively graded finite dimensional algebra over a field was treated by Wilson in $[\mathbf{1 0}]$.

Proof OF THEOREM A. Assuming that $R$ is Nakayama filtered via an $R$ filtered category $A$, let $E$ be one of the indecomposable injective objects $E_{1}, \ldots, E_{n}$ in $A$ with $\operatorname{codeg} E=c$ and let

$$
0 \leftarrow E \leftarrow Q_{0} \stackrel{f_{0}}{\longleftarrow} Q_{1} \stackrel{f_{1}}{\longleftarrow} \cdots
$$

be a minimal projective resolution of $E$ in $A$.

Since by (F2) application of $\psi$ to the above exact sequence will yield a minimal projective resolution of $\psi(E)$ in $R$-mod, all we have to show is that each $P_{i}$ occurs for at least one choice of $E \in\left\{E_{1}, \ldots, E_{n}\right\}$ as a summand of some $\psi\left(Q_{j}\right)$.

To achieve this, we will express $[E]$ as a $\mathbf{Z}((T))$-linear combination of those classes $\left[P_{i}\right]$ for which $P_{i}$ splits off in some $\psi\left(Q_{j}\right)$. Once such expansions for the $\left[E_{k}\right]$ are available, we are done: For this means that the $\mathbf{Z}((T))$-submodule of $\mathcal{G}(A)$ generated by $\left[E_{1}\right], \ldots,\left[E_{n}\right]$ is contained in the submodule generated by those $\left[P_{i}\right]$ which appear in one of the resolutions. Since the former submodule has rank $n$ by Lemma 3 , the latter cannot be generated by less than $n$ elements.

We will progressively approximate the desired expansion of $[E]$ by constructing a sequence of elements $A_{i}, R_{i}, i \geq 0$, in $\mathcal{G}(\mathcal{A})$ which satisfy the following system of equations, with $t$ running through all nonnegative integers:

$$
[E]=A_{0}+A_{1}+\cdots+A_{t}+R_{t}
$$

such that for each $s=0, \ldots, t$

$$
A_{s}=\sum_{i=1}^{n} p_{s i}\left[P_{i}\right]
$$

where the $p_{s i}$ are polynomials in $T$ and $T^{-1}$ with $\operatorname{codeg} p_{s i} \geq c+s$ and $p_{s i}=0$ if $P_{i}$ does not appear as a summand of $\psi\left(Q_{s}\right)$ in $R$-mod; of $R_{t}$ we require that

$$
R_{t}=\sum_{k=1}^{m_{t}} q_{t k}\left[B_{t k}\right]
$$

with $\bigoplus_{k=1}^{m_{t}} B_{t k}=\operatorname{Ker} f_{t}$ in $A$ and $\operatorname{codeg} q_{t k}+\operatorname{codeg} B_{t k} \geq c+t+1$.

Equation (0) results from an application of Lemma 5 to $f_{0}: Q_{0} \rightarrow E$. In fact, the equation

$$
[E]=\sum_{i=1}^{n} h_{i}\left[P_{i}\right]-\sum_{k=1}^{r} T^{u_{k}}\left[K_{k}\right]
$$


as guaranteed by Lemma 5 will yield the desired representation $[E]=A_{0}+R_{0}$ if we set $p_{0 j}=h_{j}, q_{0 k}=-T^{u_{k}}$ and $B_{0 k}=K_{k}$.

To construct $A_{t+1}$ and $R_{t+1}$ such that $(t+1)$ holds, we apply Lemma 5 to projective covers $g_{k}: P_{t k} \rightarrow B_{t k}$ with the property that

$$
f_{t+1}=\bigoplus_{k=1}^{m_{t}} g_{k}: Q_{t+1}=\bigoplus_{k=1}^{m_{t}} P_{t k} \rightarrow \operatorname{Ker}\left(f_{t}\right)
$$

We thus obtain polynomials $h_{k 1}, \ldots, h_{k n}$ in $T, T^{-1}$ and decompositions $\operatorname{Ker}\left(g_{k}\right)=$ $\bigoplus_{j=1}^{r_{k}} K_{k j}$ together with integers $u_{k 1}, \ldots, u_{k r_{k}} \geq 0$ such that

$$
\left[B_{t k}\right]=\sum_{i=1}^{n} h_{k i}\left[P_{i}\right]-\sum_{j=1}^{r_{k}} T^{u_{k j}}\left[K_{k j}\right]
$$

with

(1) $h_{k i}=0$ if $P_{i}$ is not isomorphic to a direct summand of $\psi\left(P_{t k}\right)$,

(2) $\operatorname{codeg} h_{k i} \geq \operatorname{codeg} B_{t k}$,

(3) $\operatorname{codeg} K_{k j}+u_{k j} \geq \operatorname{codeg} B_{t k}+1$,

for all $i=1, \ldots, n, k=1, \ldots, m_{t}$ and $j=1, \ldots, r_{k}$. Insertion into the above expansion of $R_{t}$ yields

$$
R_{t}=\sum_{i=1}^{n}\left(\sum_{k=1}^{m_{t}} q_{t k} h_{k i}\right)\left[P_{i}\right]+\sum_{k=1}^{m_{t}} \sum_{j=1}^{r_{k}}\left(-q_{t k} T^{u_{k j}}\right)\left[K_{k j}\right]
$$

where

$$
\begin{aligned}
\operatorname{codeg} q_{t k} h_{k i} & =\operatorname{codeg} q_{t k}+\operatorname{codeg} h_{k i} \\
& \geq \operatorname{codeg} q_{t k}+\operatorname{codeg} B_{t k} \geq c+t+1
\end{aligned}
$$

and

$$
\begin{aligned}
\operatorname{codeg} q_{t k} T^{u_{k j}}+\operatorname{codeg} K_{k j} & =\operatorname{codeg} q_{t k}+u_{k j}+\operatorname{codeg} K_{k j} \\
& \geq \operatorname{codeg} q_{t k}+\operatorname{codeg} B_{t k}+1 \geq(c+t+1)+1 .
\end{aligned}
$$

Set $p_{t+1, i}=\sum_{k=1}^{m_{t}} q_{t k} h_{k i}$. Relabeling the $K_{k j}$ as $B_{t+1,1}, \ldots, B_{t+1, m_{t+1}}$ with $m_{t+1}$ $=\sum_{k=1}^{m_{t}} r_{k}$ and the corresponding coefficients $-q_{t k} T^{u_{k j}}$ as $q_{t+1,1}, \ldots, q_{t+1, m_{t+1}}$, we arrive at $R_{t}=A_{t+1}+R_{t+1}$, as required.

Now since $\operatorname{codeg} p_{s i} \geq c+s$ for all $i$ and $s$ we can, for every $i$, sum up all the $p_{s i}, s \geq 0$, in $\mathbf{Z}((T))$ to obtain a Laurent series $p_{i}=\sum_{s \geq 0} p_{s i}$. Note that $p_{i}=0$ if $P_{i}$ is not isomorphic to a direct summand of $\psi\left(Q_{s}\right)$ for any $s$.

Finally, we conclude that

$$
[E]=\sum_{i=1}^{n} p_{i}\left[P_{i}\right]
$$

because the coefficients arising in the expansion of $A_{t}$ and $R_{t}$ via $\left[S_{1}\right], \ldots,\left[S_{n}\right]$ are Laurent series whose codegrees exceed $c+t+\min c_{i}$, where $c_{i}=\operatorname{codeg} P_{i}$.

Our concluding remark pushes an observation of Wilson $[10, p .396]$ somewhat further.

REMARK. Suppose that all indecomposable injective left $R$-modules are finitely generated. If the injective dimension of ${ }_{R} R$ is finite and $\operatorname{det} C \neq 0$, the $R$ satisfies the Generalized Nakayama Conjecture. 
ProOF. Let $0 \rightarrow R e_{i} \rightarrow U_{i 0} \rightarrow U_{i 1} \rightarrow \cdots \rightarrow U_{i t_{i}} \rightarrow 0$ be minimal injective resolutions of $R e_{i}$ in $R$-mod. In the classical Grothendieck group $K_{0}(R$-mod) this yields

$$
\left[R e_{i}\right]=\sum_{j=0}^{t}(-1)^{j}\left[U_{i j}\right],
$$

and expandingf each $U_{i j}$ into a direct sum of indecomposable components, we can express each $\left[R e_{i}\right]$ as a linear combination of the classes of those indecomposable injectives which appear as summands in at least one $U_{i j}$. But $\operatorname{det} C \neq 0$ means that $\left[R e_{1}\right], \ldots,\left[R e_{n}\right]$ are linearly independent over $\mathbf{Z}$, and thus each of the $n$ indecomposable injective modules must occur in some $U_{i j}, i=1, \ldots, n, j=0, \ldots, t_{i}$.

6. The Cartan matrix. Given an $R$-filtered category $A$, we call the following matrix $\hat{C}$ with coefficients in $\mathbf{Z}\left[T, T^{-1}\right]$ the (A-)filtered Cartan matrix of $R$ :

$$
\hat{C}=\left(\hat{c}_{i j}\right) \text {, }
$$

where $\left[P_{j}\right]=\sum_{i=1}^{n} \hat{c}_{i j}\left[S_{i}\right]$ in $\mathcal{G}(\mathcal{A})$. In other words, each $\hat{c}_{i j}$ is a polynomial $\hat{c}_{i j}=\sum_{k} c_{i j}^{(k)} T^{k}$ in $T$ and $T^{-1}$ so that $c_{i j}^{(k)}$ is the number of copies of $S_{i}$ in $\psi\left(F^{k} P_{j} / F^{k+1} P_{j}\right)$. We note that evaluation of $\hat{C}$ at $T=1$ yields the classical Cartan matrix, i.e. $C=\hat{C}(1)$. In particular, $\operatorname{det} \hat{C}=1 \mathrm{implies} \operatorname{det} C=1$.

Call $\hat{C}$ the $J$-filtered Cartan matrix if $\hat{C}$ is the filtered matrix for $A=R$-mod with the radical filtration (compare $\S 1$, Example 1 ). We start by demonstrating with two examples the superiority of the $J$-filtered Cartan matrix over the classical Cartan matrix with respect to the "finite global dimension test".

EXAMPLES. 1. Let $F$ be a field and $R$ the subring of $M_{4}(F)$ consisting of all matrices of the form

$$
\left(\begin{array}{cccc}
a & u & 0 & v \\
0 & b & 0 & 0 \\
0 & 0 & b & w \\
0 & 0 & 0 & a
\end{array}\right), \quad \text { where } a, b, u, v, w \in F
$$

The $J$-filtered Cartan matrix of $R$ is

$$
\hat{C}=\left(\begin{array}{cc}
1+T & T \\
T & 1
\end{array}\right) ;
$$

in particular, we have $\operatorname{det} \hat{C}=1+T-T^{2} \neq 1$ (which entails gl $\operatorname{dim} R=\infty$ by Theorem $\mathrm{B}$ ), whereas $\operatorname{det} C=1$.

2. [2, Example II] exhibits an example of rings $R$ and $S$ with radical cube zero having identical classical Cartan matrices, whereas gl $\operatorname{dim} R<\infty$ and $g l \operatorname{dim} S=\infty$. As it turns out, the $J$-filtered Cartan matrices still reflect finite, resp. infinite, global dimension in this case. In fact, $\operatorname{det} \hat{C}(R)=1$, whereas $\operatorname{det} \hat{C}(S) \neq 1$.

Our main goal in this section is to prove that in any of our standard settings finite left global dimension entails $\operatorname{det} \hat{C}=1$ for the correspondingly filtered Cartan matrix $\hat{C}$. For this purpose, we start again with an abstract filtration on $\mathcal{A}$.

LEMMA 6 . If $c_{i}=\operatorname{codeg} P_{i}$ for $i=1, \ldots, n$, then

$$
\operatorname{det} \hat{C}=T^{c_{1}+\cdots+c_{n}}+p,
$$

where $p \in \mathbf{Z}\left[T, T^{-1}\right]$ with $\operatorname{codeg} p>c_{1}+\cdots+c_{n}$. 
PROOF. Focus on the $j$ th column of $\hat{C}$ and note that $\hat{c}_{j j}=T^{c_{j}}+q_{j}$, where $\operatorname{codeg} q_{j}>c_{j}$, whereas $\hat{c}_{i j}>c_{j}$ for all $i \neq j$. For any nontrivial permutation $\sigma$ of $\{1, \ldots, n\}$ this gives us $\operatorname{codeg}\left(\hat{c}_{1 \sigma(1)} \cdots \hat{c}_{n \sigma(n)}\right) \geq c_{1}+\cdots+c_{n}+2$, and our claim follows.

To establish our main theorem, we intend to employ a trick similar to that of $\S 5$, and again we will need the morphisms in the relevant projective resolutions to be well behaved.

Call $R$ Cartan filtered if $R$ admits a filtered $R$-category $A$ in which each $S_{i}$ has a minimal projective resolution

$$
0 \leftarrow S_{i} \stackrel{f_{i 0}}{\longleftarrow} Q_{i 0} \stackrel{f_{i 1}}{\longleftarrow} Q_{i 1} \leftarrow \cdots
$$

such that all direct summands of the epimorphisms $f_{i j}: Q_{i j} \rightarrow \operatorname{Im}\left(f_{i j}\right)$ respect the filtration.

If $R$ is Cartan filtered, then, in particular, the projective covers $P_{i} \rightarrow S_{i} \rightarrow 0$ respect the filtration, whence $\operatorname{codeg} P_{i}=0$ for all $i$ (Lemma 5). Consequently, $\hat{C}$ is a matrix in $M_{n}(\mathbf{Z}[T])$ with $\operatorname{det} \hat{C}=1+p$, where $\operatorname{codeg} p>1$.

THEOREM B. If $R$ is a Cartan filtered ring of finite left global dimension, then $\operatorname{det} \hat{C}=1$, and in particular, the determinant of the classical Cartan matrix equals 1 .

COROLLARY (see [10] for the case of a finite dimensional, positively graded algebra). (1) If $R$ satisfies $J^{3}=0$ or is positively graded, then finiteness of the left global dimension of $R$ implies $\operatorname{det} \hat{C}=1$. (Here $\hat{C}$ is the $J$-filtered matrix in the former case, and filtered with respect to the grading in the latter.)

(2) Suppose that $R$ is left serial and $\hat{C}$ the $J$-filtered Cartan matrix. Then $R$ has finite left global dimension if and only if $\operatorname{det} \hat{C}=1$.

PROOF. Compare with $\S 4$ to see that all the rings under discussion are Cartan filtered. For (2) use Theorem B and the fact that $\operatorname{det} \hat{C}=1 \operatorname{implies} \operatorname{det} C=1$, while the latter condition entails finite left global dimension for left serial rings by [2, Theorem 6].

PROOF OF THEOREM B. Suppose that $A$ meets the requirements for a Cartan filtration and that $1 \mathrm{gl} \operatorname{dim} R<\infty$. Condition (F2) then guarantees that the minimal projective resolutions of the $S_{i}$ in $A$ terminate; denote them by

$$
0 \leftarrow S_{i} \stackrel{f_{0}}{\longleftarrow} Q_{i 0} \stackrel{f_{1}}{\longleftarrow} Q_{i 1} \stackrel{f_{2}}{\longleftarrow} \cdots \leftarrow Q_{i m_{i}} \leftarrow 0 .
$$

Noting that

$$
0=\operatorname{codeg} S_{i}=\operatorname{codeg} Q_{i 0} \leq \operatorname{codeg}\left(\operatorname{Ker} f_{0}\right)=\operatorname{codeg} Q_{i 1} \leq \cdots
$$

and proceeding as in the proof of Theorem $\mathrm{A}$, we find polynomials $d_{j i} \in \mathbf{Z}[T]$ such that

$$
\left[S_{i}\right]=\sum_{j=1}^{n} d_{j i}\left[P_{j}\right]
$$


in $\mathcal{G}(A)$. By the defintion of the $\hat{c}_{i j}$ we infer

$$
\begin{aligned}
{\left[S_{i}\right] } & =\sum_{j=1}^{n} d_{j i} \sum_{k=1}^{n} \hat{c}_{k j}\left[S_{k}\right] \\
& =\sum_{k=1}^{n}\left(\sum_{j=1}^{n} \hat{c}_{k j} d_{j i}\right)\left[S_{k}\right]
\end{aligned}
$$

that is, if $\hat{D}=\left(d_{i j}\right)$, then $\hat{C} \hat{D}=I_{n}$, the identity matrix in $M_{n}(\mathbf{Z}[T])$. Thus $\operatorname{det} \hat{C}$ is a unit in $\mathbf{Z}[T]$. But since $\operatorname{det} \hat{C} \neq-1$ by Lemma 6 , the proof is complete.

REMARK. In a forthcoming paper we will show that for $J^{2}=0$ we have

$$
\operatorname{det} \hat{C}=1 \Leftrightarrow \operatorname{lgl} \operatorname{dim} R<\infty,
$$

whereas for $J^{3}=0$, the $J$-filtered Cartan matrix alone no longer suffices to distinguish between finite and infinite global dimension.

Green, Gustafson and Zacharia [6] have recently shown that 1 gl $\operatorname{dim} R \leq 2 \mathrm{im}$ plies $\operatorname{Ext}_{R}^{1}(S, S)=0$ for an arbitrary simple left $R$-module $S$. It is apparently an open question whether, more generally, $\operatorname{lgl} \operatorname{dim} R<\infty$ entails the same conclusion. For Cartan filtered rings we have the following

COROLlaRY. If $R$ is Cartan filtered by the radical filtration (e.g. if $J^{3}=0$ or $R$ is left serial) and $\operatorname{lgl} \operatorname{dim} R<\infty$, then $\operatorname{Ext}_{R}^{1}(S, S)=0$ for every simple left $R$-module $S$.

PROOF. In Lemma 6 , we have $c_{i}=0$ for all $i$, and the argument we give there shows that the polynomial $\operatorname{det} \hat{C}$ has a nonzero linear term if and only if this is true for at least one diagonal coefficient $\hat{c}_{i i}$ of $\hat{C}$. On the other hand, $\operatorname{Ext}_{R}^{1}\left(S_{i}, S_{i}\right) \neq 0$ clearly forces a linear term upon $\hat{c}_{i i}$, a situation which is excluded by the condition $\operatorname{det} \hat{C}=1$.

ADDED IN PROOF. In connection with the concluding remark of $\S 5$ : Y. Iwanaga [Comm. Algebra 7 (1979), 393-414] had already proved the (GNC) for noetherian rings of finite left and right injective dimension.

\section{REFERENCES}

1. M. Auslander and I. Reiten, On a generalized version of the Nakayama Conjecture, Proc. Amer. Math. Soc. 52 (1975), 69-74.

2. W. D. Burgess, K. R. Fuller, E. R. Voss and B. Zimmermann-Huisgen, The Cartan matrix as an indicator of finite global dimension for artinian rings, Proc. Amer. Math. Soc. 95 (1985), 157-165.

3. V. P. Camillo and K. R. Fuller, On graded rings with finiteness conditions, Proc. Amer. Math. Soc. 86 (1982), 1-5.

4. S. Eilenberg, Algebras of cohomologically finite dimension, Comment. Math. Helv. 28 (1958), 310-319.

5. R. Gordon and E. Green, Graded Artin algebras, J. Algebra 26 (1982), 111-137.

6. E. Green, W. Gustafson and D. Zacharia, On Artin rings of global dimension two, J. Algebra 92 (1985), 375-379.

7. K. Morita and H. Tachikawa, On QF-3 rings (unpublished).

8. B. J. Mueller, The classification of algebras by dominant dimension, Canad. J. Math. 20 (1968), 398-409. 
9. H. Tachikawa, Quasi-Frobenius rings and generalizations, Lecture Notes in Math., vol. 351, Springer-Verlag, Berlin and New York, 1973.

10. G. Wilson, The Cartan map on categories of graded modules, J. Algebra 85 (1983), 390398.

11. D. Zacharia, On the Cartan matrix of an Artin algebra of global dimension two, J. Algebra 82 (1983), 353-357.

Department of Mathematics, University of Iowa, Iowa City, Iowa 52242

FAKULTÄT FÜR MATHEMATIK UND INFORMATIK, UNIVERSITÄT PASSAU, 8390 PASSAU, BundesRepublik DeUtschland 